\title{
Epiallelic Variation in Arabidopsis thaliana
}

\author{
R.C. O’Malley and J.R. EckeR \\ Genomic Analysis Laboratory, The Salk Institute for Biological Studies, La Jolla, California 92037 \\ Correspondence: ecker@salk.edu
}

\begin{abstract}
Genotype is the primary determinate of phenotype. During the past two decades, however, there has been an emergent recognition of the epigenotype, a separate layer of heredity distinct from the primary DNA sequence that can have profound effects on phenotype. The epigenotype is a collection of chemical modifications to the DNA and nucleosomes in conjunction with noncoding RNA transcripts, and together these epigenetic marks act as a potent and expansive regulatory system for controlling gene expression. In this review, we discuss our current understanding of variation in epigenotype in the model plant Arabidopsis and how allelic differences attributable to epigenetic changes, or epialleles, can affect phenotype. We discuss examples of epialleles that have been created in the laboratory and others that have been identified in natural populations, because these two models provide complementary information regarding the genetic pathways, mechanisms of transmission, and biological and evolutionary context for the role of the epigenotype in phenotypic variation.
\end{abstract}

Arabidopsis thaliana is found over a large swath of Eurasia and parts of North Africa, exposing individual populations to an array of biotic and abiotic challenges (Koornneef et al. 2004). The significant phenotypic variation that exists among regional populations of this species is reflective of the need to cope with a diverse spectrum of environmental variables (Koornneef et al. 2004). Wild Arabidopsis isolates from specific geographic locations, known as accessions, have proved to be a valuable resource for studying phenotypic variation, shedding light on how a plant species diverges in response to varying degrees of isolation and adaptation to local environments (Fournier-Level et al. 2011; Hancock et al. 2011).

Although largely similar with regard to basic anatomy, Arabidopsis accessions show phenotypic differences in important developmental traits such as leaf shape, growth rate, biomass, and flowering time (Weigel 2012). Perhaps even more striking is the enormous variation observed in response to specific biotic and abiotic challenges. Dramatic phenotypic variations are observed when accessions are exposed to various challenges such as hormones, bioactive small molecules, metals, salts, light, heat, cold, moisture, and pathogens, all of which are environmental variations likely to be encountered by a widely dispersed population (Weigel 2012). Recent sequencing of Arabidopsis accessions genomes reveals substantial intraspecific sequence divergence in the form of single-nucleotide polymorphisms (SNPs) and both small and large insertions and deletions (Ossowski et al. 2008; Santuari et al. 2010; Cao et al. 2011; Gan et al. 2011; Schneeberger et al. 2011). Many genes are affected in terms of coding sequence, and significant transcriptional variation is observed between the accessions, providing an initial blueprint of the evolutionary forces shaping transcriptome variation, a primary wellspring of phenotypic divergence (Cao et al. 2011; Gan et al. 2011).

Differences in genotype were once viewed as the sole cause of natural phenotypic variation, but in the past two decades it has been recognized that epigenetic variation, consisting of heritable modifications not directly encoded in the nucleotide sequence, may provide a distinct mechanism for inter- and intraspecific variation (Paszkowski and Grossniklaus 2011). Heritable epigenetic marks such as cytosine methylation, histone modifications, histone variants, and small RNA have all been shown to play a role in regulation of gene expression (Law and Jacobsen 2010; Roudier et al. 2011). Alleles variant in transcriptional regulation resulting from epigenetic differences are commonly referred to as epialleles. Although only a few examples of natural phenotypic variation caused by epialleles have been identified to date, there is evidence of substantial epigenetic variation among Arabidopsis accessions, suggesting the potential for a major contribution of epiallelic divergence on phenotype (Groszmann et al. 2011; Greaves et al. 2012b; Shen et al. 2012).

Cytosine DNA methylation, the addition of a methyl group to the fifth position of cytosine in nuclear DNA, is one of the best characterized of these epigenetic modifications and has been shown to have profound effects on regulation of gene and transposon transcription (Matzke et al. 2007; Slotkin and Martienssen 2007). Cytosine DNA methylation is also the most described epigenomic variation with regard to natural and artificially induced epialleles in plants (Richards 2011). Furthermore, with recent developments in DNA sequencing, single-basepair-resolution whole-genome methylome maps of $\mathrm{Ara}$ bidopsis can be generated quickly and relatively inexpensively, promising future enrichment of the annotation of DNA methylation in many Arabidopsis accessions (Cokus et al. 2008; Lister et al. 2008). As a result, in 
this review, we largely focus on epialleles associated with variation in DNA methylation, although other epigenetic marks are discussed when relevant. We discuss the generation of artificial epialleles that have proved to be powerful tools for studying the genetic pathways and mechanisms controlling DNA methylation; artificial epialleles have proved to be valuable model systems to inform our understanding of naturally occurring epialleles. The second half of the review offers examples of naturally occurring epialleles and epigenomic variation with a focus on phenotypes, associated sequence features, transmission, heritability, and the complex interplay that can exist between genotype and epigenotype.

\section{DNA METHYLATION IN ARABIDOPSIS}

The single-base-pair resolution possible with next-generation sequencing allowed for direct analysis of the extent and frequency of methylation for each of the targeted cytosine contexts in Arabidopsis (Cokus et al. 2008; Lister et al. 2008). Overall, $\sim 6 \%$ of cytosines were found to be methylated, in close agreement with bulk methylcytosine content measured by high-pressure liquid chromatography (HPLC) (Rozhon et al. 2008). In eukaryotes, cytosine methylation is catalyzed by DNA methyltransferase. Arabidopsis has three classes of these genes: DNA methyltransferase 1 (MET1), chromomethylase 3 (CMT3), and domain-rearranged methyltransferase 1 and 2 (DRM1/2) (Law and Jacobsen 2010). Each DNA methyltransferase is regulated by a distinct genetic pathway and targets a specific sequence context (Law and Jacobsen 2010).

MET1, a homolog of the mammalian methyltransferase DNMT1, deposits DNA methylation at the dinucleotide CG accounting for $\sim 55 \%$ of methylated sites in Arabidopsis (Finnegan et al. 1996; Ronemus et al. 1996; Kankel et al. 2003; Saze et al. 2003; Cokus et al. 2008; Lister et al. 2008). MET1 may target hemimethylated DNA that is present after DNA replication, allowing for faithful propagation of methylation to the symmetric CG site on the newly synthesized daughter strand. This mechanism has been demonstrated in mammalian systems, where the UHRF1 (ubiquitin-like-containing, PHD, ring finger) protein binds to both DNMT1 and hemimethylated DNA, providing an interface between the DNA methyltransferase and target DNA (Bostick et al. 2007; Sharif et al. 2007; Hashimoto et al. 2008; Kim et al. 2009). VIM1, an Arabidopsis homolog of UHRF1, is important for maintenance of CG methylation and has been shown to bind methylated DNA, but not exclusively hemimethylated $\mathrm{CG}$, suggesting that it may play a similar, although perhaps not biochemically identical, function as UHRF1 (Woo et al. 2007; Kraft et al. 2008). UHRF1 has also been shown to bind specific histone methyltransferases and deacetylases, suggesting a direct association between DNA methylation and histone silencing marks (Kim et al. 2009). A null mutation in the Arabidopsis HDA6, a histone deacetylase, results in reduced $\mathrm{CG}$ methylation and derepression of many of the same genes observed in the met $1-3$ null mutation
(Aufsatz et al. 2002, 2007; To et al. 2011). MET1 and HDA6 have been shown to interact directly in vivo and in vitro, supporting a model that colocalization of MET1 and histone modifiers at targeted loci is important in CG methylation in Arabidopsis (Liu et al. 2012).

CMT3, a plant-specific DNA methyltransferase, catalyzes DNA methylation in the CHG context and accounts for $\sim 22 \%$ of all methylated sites in Arabidopsis (where $\mathrm{H}=\mathrm{A}, \mathrm{C}$, or T) (Bartee et al. 2001; Lindroth et al. 2001; Cokus et al. 2008; Lister et al. 2008). Genetic evidence indicates that the establishment of the CHG methylation is dependent on the histone marks H3K9me2 and $\mathrm{H} 3 \mathrm{~K} 27 \mathrm{me}$ and that CMT3 can directly bind to H3K9me2 (Lindroth et al. 2004). Consistent with this, CHG methylation is dependent on the activity of a family of H3K9 methyltransferases (KRY/SUVH4, SUVH5, and SUVH6) and CHG methylation is associated with H3K9me2 genome wide (Jackson et al. 2002; Malagnac et al. 2002; Bernatavichute et al. 2008). Furthermore, KRY has been shown itself to bind directly to methylated DNA, with a preference for $\mathrm{CHG}$ and $\mathrm{CHH}$ contexts, providing a potential reinforcing loop between H3K9me2 and CHG methylation (Johnson et al. 2007). The loss of a H3K9 demethylase, IBM1, also has a profound effect as many genes become ectopically methylated in the CHG context in the ibm 1 null, suggesting that this enzyme is actively demethylating $\mathrm{H} 3 \mathrm{~K} 9$ from many genes and preventing CHG methylation (Inagaki et al. 2009).

The third class of DNA methyltransferase, DRM1 and DRM2, methylate cytosines in the asymmetric $\mathrm{CHH}$ sequence context that comprises $22 \%$ of all methylated sites (Cao and Jacobsen 2002; Cokus et al. 2008; Lister et al. 2008). DMR2 is directed to target loci by small RNAs (smRNAs) and a specific RNA interference pathway known as RNA-directed DNA methylation (RdDM) (Chan et al. 2004). A number of mutants have been discovered in this pathway, allowing for extensive epistatic and molecular analysis (Law and Jacobsen 2010). The general model consistent with the current data begins with expression of a primary transcript by the DNA polymerases Pol IV, a plant-specific variant of Pol II (Herr et al. 2005; Kanno et al. 2005; Onodera et al. 2005). This transcript is then converted into double-stranded RNA by the RNA-directed RNA polymerase RDR2, processed by the Dicer-like DCL3, and the resultant short interfering RNA (siRNA) is loaded into the Argonaut AGO4 (Zilberman et al. 2003; Chan et al. 2004; Xie et al. 2004). AGO4siRNA then directs a protein complex that includes the DNA methyltransferase DRM2 to specifically target methylation to a locus via siRNA hybridization to a "guide" transcript generated by Pol V, a distinct DNA polymerase variant to the one that synthesized the primary transcript used to generate siRNA (Henderson and Jacobsen 2007; Matzke et al. 2009). As is the case for CMT3, DRM2 also requires two SUVH methylcytosine binding proteins for $\mathrm{CHH}$ methylation activity (Johnson et al. 2008).

RdDM targeted loci are characterized by dense methylation in all sequence contexts, not just $\mathrm{CHH}$, and show a 
high incidence of overlap with siRNA, particularly of the 24-bp size class (Lister et al. 2008). Thus, although the establishment of methylation by RdDM at newly inserted transgenes has been shown to be dependent on the presence of DRM2 and an intact siRNA pathway, the other two methyltransferases must be recruited to these sites as well, because methylation is found in all sequence contexts (Cao and Jacobsen 2002; Chan et al. 2004). It has yet to be clearly established by what mechanisms all three pathways converge at these loci, and this will certainly be an important future direction of study. It is interesting to note, however, that several of the DNA methylation "readers," VIM1-3 and the SUVH2-9 family members, which have been shown to have effect-specific DNA methylation context, show much lower specificity with regard to binding of specific methylcytosine contexts (Woo et al. 2007, 2008; Johnson et al. 2008). It will be interesting to see whether recruitment of histone methyltransferases and deacetylases to methylated DNA may provide a potential "cross talk" between the distinct DNA methyltransferase pathways. The potential roles for histone modifiers in RdDM is an active field of investigation (Saze et al. 2012).

Different RdDM loci show substantial heterogeneity in the relative contribution of specific genes in the methyltransferase, siRNA, and histone-modifying pathways. The variation in the role of methyltransferases in silencing at different loci can clearly be seen by the fact that, of the more than 500 genes that are derepressed in the met 1 and $d d c$ backgrounds, $<10 \%$ are up-regulated in both (based on a comparison using data from http://neomorph. salk.edu/ julian/clusters_simple.php) (Lister et al. 2008). Different histone methyltransferases have similarly been observed to have loci-specific effects on DNA methylation, because different members of the SUVH3 -9 show overlapping effects at some loci and independent effects at others. In the RNAi (RNA interference) pathway, a number of the key genes are members of gene families and in some cases mutants in specific paralogs affect some RdDM loci more than others. For example, although AGO4 is required to target RdDM at many loci (Zilberman et al. 2003, 2004), AGO6 has also been shown to contribute to RdDM in a partly redundant manner at a subset of loci (Zheng et al. 2007). Additionally, whereas a Pol V mutant has been shown to disrupt RdDM at several loci (Wierzbicki et al. 2009), potentially owing to loss of the scaffolding transcript, there is evidence that Pol II may contribute to generation of scaffold transcripts and/or is involved in the establishment or stabilization of the interaction of AGO-siRNA at some low-copynumber loci (Zheng et al. 2009). Further integration of multiple levels of epigenomic information in various null mutants may provide a clearer picture of the contribution of different methyltransferase, RNAi, and histone-modification pathways.

DNA transposons and retrotransposons are heavily targeted by RdDM in plants, and a primary function of this epigenetic regulation appears to be transcriptional silencing to limit transposon mobilization (Law and Jacobsen 2010). One study found that $74 \%$ of annotated transposons are methylated and the remaining $26 \%$ of unmethylated transposon were enriched for short, highly degenerate relics, many of which might be expected to have lost transcriptional and transposable competency (Ahmed et al. 2011). Transcriptome sequencing of met 1 has shown that hundreds of transposon/pseudogenes are derepressed after loss of CG methylation (Lister et al. 2008). Furthermore, mobilization of several classes of transposons have been seen in metl as well as in other RdDM deficient backgrounds, clearly demonstrating a protective role for RdDM silencing of transposons on genomic integrity (Kato et al. 2004; Mirouze et al. 2009; Reinders et al. 2009; Tsukahara et al. 2009; Matsunaga et al. 2012). Our understanding of how transposons are targeted for RdDM is still work in progress, although several features common to transposons have been proposed to have a role in attracting RdDM, including enrichment for repeat sequences, high copy number, convergent and sense-antisense transcription, and high expression levels observed when silencing is derepressed and transposition is occurring (Slotkin and Martienssen 2007).

Gene expression is also affected by RdDM, frequently resulting in partial or complete transcriptional silencing. In both the met 1 and $d d c$ background, hundreds of genes are up-regulated, significantly changing the transcriptome of these mutants (Lister et al. 2008). In the following sections, we explore artificially induced epialles and discuss some of the lessons that they have taught us regarding phenotypic effects, genetic pathways, stability, and unique properties of RdDM on gene regulation.

\section{HYPOMETHYLATION MUTANTS AND INDUCED EPIALLELES}

Segregation analysis of a met 1 loss-of-function mutant shows that homozygous individuals are recovered at only $2 \%$ of the expected frequency, indicating that MET 1 activity is critical in early embryo development (Saze et al. 2003). This may be attributable, at least in part, to the demonstrated role of methylation in controlling imprinted genes. Surprisingly, however, the met 1 null plants that do germinate do not display gross morphological defects because plants appear to tolerate the complete loss of CG methylation. However, a number of stable heritable phenotypes segregate independently of the met 1 allele (Reinders et al. 2009). Phenotypically, the triple mutant $d r m 1 d r m 2 \mathrm{cmt} 3$ (hereafter, $d d c$ ) is less severe than the met1, although reduced stature and leaf curling is observed in this background (Cao and Jacobsen 2002; Chan et al. 2006a). DDM1, a chromatin remodeling protein, is not directly responsible for deposition of methyl groups, yet a null $d d m 1$ mutation causes a $70 \%$ drop in global methylation, primarily owing to the loss of $\mathrm{CG}$ methylation, similar to what is seen in met1 (Vongs et al. 1993; Jeddeloh et al. 1998, 1999). ddml has some of the same phenotypic effects as metl, including late flowering and reduced stature that appear to be attributable to some of the same hypomethylated epialleles 
(Johannes et al. 2009; Reinders et al. 2009). Unlike met1, however, $d d m 1$ plants require several generations of propagation to fully establish the hypomethylated state, and likewise the hypomethylated phenotypes become more pronounced in later generations of inbred plants (Jeddeloh et al. 1998, 1999; Brzeski and Jerzmanowski 2002).

Stable, heritable phenotypes in these hypomethylated backgrounds have been mapped by genetic linkage. These induced epialleles have been instrumental in determining the components of the genetic pathway and identifying sequence features associated with RdDM. One extensively studied induced epimutant is in the FLOWERING WAGENINGEN (FWA) gene, a homeodomain transcription factor. In wild-type plants, $F W A$ is an imprinted gene expressed only by the maternal allele and only in the developing endosperm (Kinoshita et al. 2004). The epiallele fwal, on the other hand, is unmethylated and expressed ectopically in vegetative tissues, resulting in a late flowering phenotype (Soppe et al. 2000; Kankel et al. 2003; Saze et al. 2003). A hypomethylated fwa allele has been found to be responsible for the late flowering phenotype seen in both $d d m 1$ and metl backgrounds as well as the original fwal allele identified from a chemically mutagenized population (Soppe et al. 2000; Kankel et al. 2003; Saze et al. 2003).

Whereas the late flowering phenotype is likely to be an artifact of ectopic expression, fwa has been a valuable tool for identifying many of the components of the RdDM pathway. Insertion of an additional copy of an FWA sequence by Agrobacterium-mediated transformation into a wild-type plant results in silencing of the newly introduced transgene (Cao and Jacobsen 2002). However, when RdDM is compromised, the transgene remains unmethylated and expressed, resulting in the late flowering phenotype. Screening for mutants incapable of silencing the FWA transgene in this manner allowed for identification of the de novo methyltransferases DRM2 and provided the first demonstration of a role for RNAi silencing genes in RdDM (Cao and Jacobsen 2002; Chan et al. 2004).
In addition to its use as a reporter for mutants in the RdDM pathway, the $F W A$ transgene system has been an informative model for understanding the types of primary sequence features responsible for attracting RdDM. The methylated region in wild-type FWA overlaps with an annotated retrotransposon of the SINE (short interspersed nuclearelement) family (Chan et al. 2006b; Kinoshita et al. 2007). Constructs containing truncated versions of the FWA gene and upstream sequences have been used to demonstrate that the SINE is necessary and sufficient for RdDM targeting (Chan et al. 2006b; Kinoshita et al. 2007). Because the majority of the SINE sequence consists of a pair of direct repeats, a common target for RdDM, a transgene that contained only the tandem repeat sequence was also tested and found to be a sufficient target for methylation (Chan et al. 2006b; Kinoshita et al. 2007). Furthermore, when the two direct repeats were reduced to just a single copy of each, no methylation occurred, indicating that repeat-character and not the intrinsic sequence that they encode is responsible for the targeting of RdDM (Chan et al. 2006b; Kinoshita et al. 2007).

Gene silencing as a direct consequence of a proximal repeat sequence has been observed in a number of other induced epialleles. One well-characterized example is an epiallele of Suppressor of $d d c(S D C)$, an F-box protein, which was identified as the cause of a dwarf phenotype in the $d d c$ background (Henderson and Jacobsen 2008). In wild-type plants, $S D C$ is methylated in a $2-\mathrm{kb}$ region of its promoter and no expression of the gene can be detected at any stage of development (Henderson and Jacobsen 2008). In the $d d c$ background, this promoter methylation is lost and the gene is expressed in all tissues (Henderson and Jacobsen 2008). As is the case with $F W A$, methylation of the $S D C$ promoter is associated with a tandem direct repeat, further indicating that repeat sequences by themselves can be sufficient for RdDM targeting (Fig. 1). Unlike $F W A$, however, where methylation is largely restricted to the tandem repeats region, methylation and siRNA production in the $S D C$ promoter is spread many
FWA

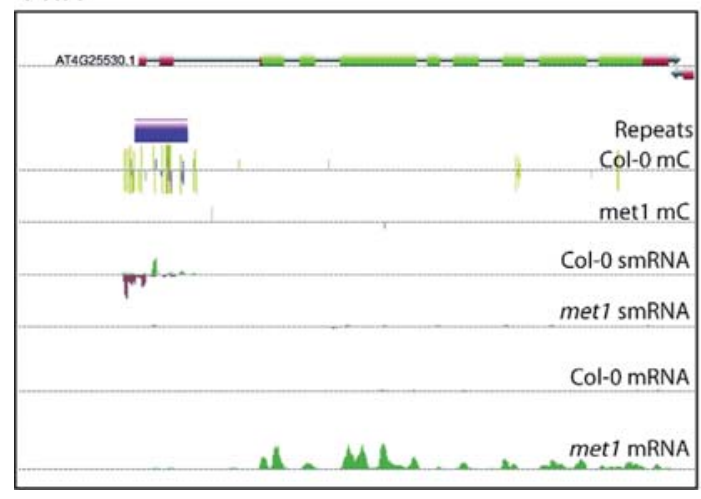

$S D C$

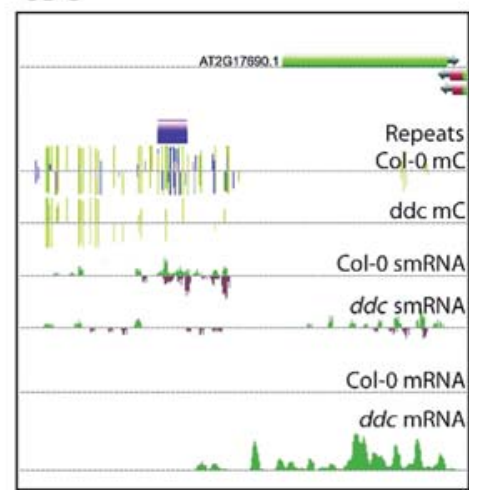

Figure 1. FWA and SDC in wild-type and hypomethylated mutant backgrounds. The FWA gene is shown with tracks containing the methylcytosines (CG, tan; CHG, blue; CHH, red), smRNA, and mRNA (messenger RNA) maps of the Col-0 and met 1 background (Lister et al. 2008). The SDC gene is shown with the same tracks for the Col-0 and $d d c$ background. The purple bar in the "Repeats" track represents a region containing direct repeats. 
hundreds of base pairs upstream of and downstream from the repeats (Henderson and Jacobsen 2008). The spreading observed into SDC is lost in the metl background, although the methylation at the repeat site is retained, suggesting a possible role for MET1/CG methylation in spreading at this locus (Henderson and Jacobsen 2008). Interestingly, it was observed that siRNA and DNA methylation are capable of spreading independently from each other based on experiments with a set of RNAi and DNA methyltransferase null mutants (Henderson and Jacobsen 2008). In a bioassay using a two-component silencing system where one locus with a hairpin was used to target a second transgenic site, Daxinger et al. (2009) observed that spreading of $\mathrm{RdDM}$ at the target site required a functional Pol IV, RDR2, and DCL3, suggesting the possibility that a Pol IV primary transcript was generated extending beyond the sequence shared by the hairpin and target sites (Daxinger et al. 2009). A deeper understanding of the genetic pathways and the underlying sequences that promote or contain spreading may be important for understanding natural variation in epialleles, because this commonly observed phenomenon could provide a mechanism by which methylation at repeats and transposons can impinge on regulatory regions in neighboring genes.

Transmittance of an epiallelic state from an endogenous gene to an unlinked homologous sequence demonstrates another important feature of epialleles that distinguishes them from genetic mutations. Methylation of the $F W A$ transgene is strongly affected by the epiallelic state of the endogenous $F W A$. When wild-type plants are transformed with $F W A$, the transgene is always silenced, whereas transformation of a plant containing the endogenous fwal epiallele results in silencing only $7 \%$ of the time (Chan et al. 2006b). This dependency of methylation at the transgene as a function of the epigenetic state of the endogenous gene strongly suggests that a direct epigenetic interaction exists among these unlinked loci. One model consistent with this would be that the siRNA produced at the endogenous copy is required for trans-RdDM targeting of the transgene and that the fwal has a reduced siRNA level, an effect frequently observed at many hypomethylated sites (Lister et al. 2008). However, a northern blot of the siRNA specific to fwal, an epiallele isolated from a chemically mutagenized population, did not show a significant reduction in siRNA levels compared to the wild-type $F W A$ (Chan et al. 2006b). It is worth noting, however, that the same blot showed no reduction in siRNA from an fwa generated in the metl background, whereas another study had shown by latent nuclear antigen (LNA) pull-down experiments that the siRNA level in met 1 decreases substantially (Lippman et al. 2004). A similar substantial decrease in met 1 siRNA is also evident in bisulfite and smRNA sequencing maps of met1 (Fig. 1) (Lister et al. 2008). It might be possible, therefore, that the smaller dynamic range of the northern blot failed to capture a small, but potentially significant, decrease in the fwa siRNA that could explain the reduced RdDM targeting in trans. However, it is impossible to rule out that some other unidentified epigenetic state of the fwal unrelated to its siRNA levels was responsible for limiting its ability to transmit methylation to the endogenous fwa copy. Understanding whether a simple siRNA dosage effect or some other unidentified mechanism dictates the efficacy of trans RdDM of the transgenic FWA could be very relevant for studying naturally occurring epialleles where trans RdDM is frequently observed (Greaves et al. 2012a).

In a similar vein, it has been observed that maintenance of a hypomethylated epiallele is strongly dependent on how much of the wild-type methylation and siRNA are retained in the hypomethylated background. In a study of remethylation of loci in the $d d m 1$ background, after reintroduction of a wild-type DDM1 by outcrossing, it was observed that loci that showed higher retention of siRNA and methylation were more likely to remethylate than loci where most of the siRNA and methylation were lost in the hypomethylated parent (Teixeira et al. 2009). Although not examined in this study, fwa generated in $d d m 1$ and met 1 show significantly different likelihood to be remethylated after outcrossing, and this may be caused by the degree of siRNA/methylation depletion seen at the fwa loci in each background. The met l-generated fwa shows a much greater depletion of siRNA levels compared to the $d d m$ 1-generated fwa (Lippman et al. 2004), but in both backgrounds in the first generation after an outcross, the fwa remains unmethylated. However, after three additional generations, the $d d m 1$-generated $f w a$ was silenced in most lines, whereas in the met1 background, in contrast, no silencing was observed (Johannes et al. 2009; Reinders et al. 2009). This suggests that the degree of hypomethylation and related loss of siRNA in the two backgrounds may be an important determinate of the stability of their respective fwa epialleles. Additionally, it supports a model where an siRNA dose dependency may be important for the stability and reestablishment of RdDM.

These induced epialleles have been informative models, providing a tractable experimental system for exploring the pathways and sequence requirements involved in cis and trans silencing. Much of what has been observed in these systems has also been observed in several naturally occurring epialleles that have been described during the past decade. In addition to providing confirmation of many of the phenomena observed in the induced epialleles, these natural epialleles provide concrete examples of how epigenetic and structural variation in divergent accessions can interact to establish cis and trans methylation, ultimately effecting traits in a non-Mendialian fashion.

\section{NATURALLY OCCURRING EPIALLELES}

One well-characterized example of a natural epiallele occurs in the phosphoribosylanthranilate isomerase (PAI) gene family (Fig. 2). In Col-0, as well as the majority of other accessions examined, PAI is encoded by a threemember gene family (Bender and Fink 1995). However, in some accessions, including Wassilewskija (Ws), a fourth paralog, $P A I 4$, is found in a tail-to-tail arrangement 


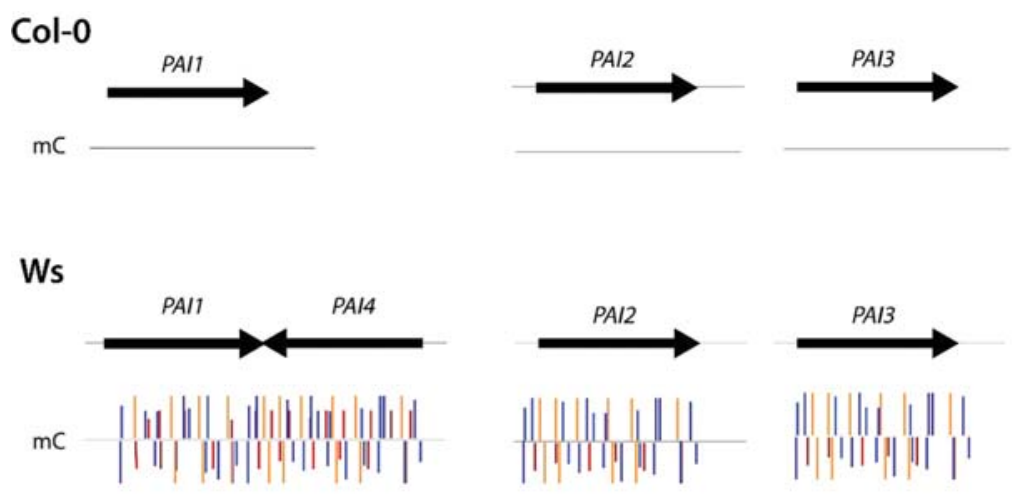

\section{Col- $0 \times$ Ws}

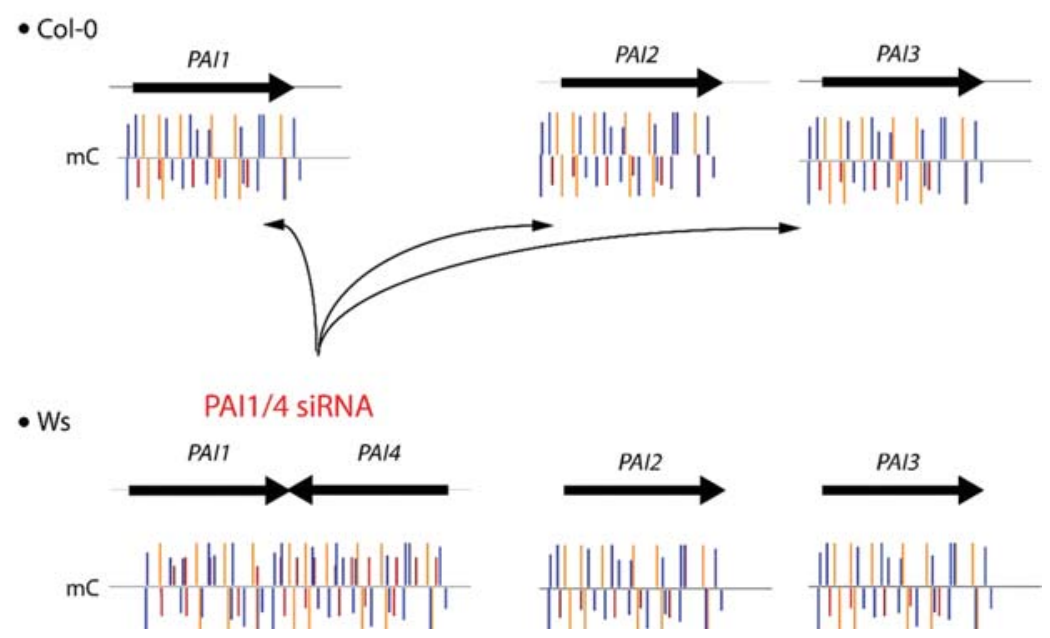

Figure 2. Effect of $P A I 1 / 4$ rearrangement on RdDM in trans. The three-membered phosphoribosylanthranilate isomerase (PAI) gene family in Col-0 is depicted with the corresponding image showing the absence of RdDM in the track labeled " $m$ C." The Ws accession contains a fourth copy (PAI4) in a tail-to-tail arrangement with PAI1, and all four genes in this accession are targeted by RdDM. The Col- $0 \times$ Ws shows the consequence of an F1 cross between Ws and Col-0 that results in RdDM targeting of the Col-0 PAI gene family from siRNA produced at the Ws PAII/4 direct repeat.

with PAIl (Bender and Fink 1995). The large inverted repeat resulting from this $P A I 1 / 4$ rearrangement in Ws results in the targeting of RdDM over the entire length of both genes and in lower expression of PAIl relative to Col-0 (Bender and Fink 1995). In addition to silencing at $P A I 1 / 4$, the unlinked $P A I 2$ and $P A I 3$ genes are also methylated in Ws but not in Col-0 (Bender and Fink 1995). When Ws was crossed to Col-0, all three unmethylated copies of the Col-0 PAI genes become methylated in the $\mathrm{F}_{1}$. In $\mathrm{F}_{2}$ segregants that lose the Ws, PAIl / 4 methylation of the PAI2 and PAII3 genes also lost, demonstrating a direct requirement of the inverted repeat for maintaining the trans RdDM (Bender and Fink 1995).

Although the inverted repeat in PAII/4 is attributable to a gene duplication, a similar requirement for a repetitive sequence for RdDM targeting as is seen in FWA and $S D C$ suggests that structural variation in repeat sequences may be a powerful trigger for RdDM. Gene duplications and rearrangements are also common in plant genome evolution, so this type of feature may also be a common mechanism by which epialleles are established.
Furthermore, $P A I$ illustrates how once a rearrangement is established, RdDM in trans may influence transmission of this epiallelic state to close paralogs. However, at least in this case, genetic segregation of the triggering repeat results in loss of the epiallelic state, indicating that at some epialleles, the genetic and epigenetic states are linked.

Naturally occurring epialleles with visible phenotypic consequence have been identified in other plants including the tomato Colorless non-ripening ( $\mathrm{Cnr}$ ) locus, peloric in Linaria and paramutation of the Booster $\left(b^{\prime}\right)$ locus in maize (Cubas et al. 1999; Stam et al. 2002; Manning et al. 2006). Although all three provide interesting examples of natural epimutations, the $b^{\prime}$ locus is the best characterized in terms of the genetic requirements and sequence features associated with the establishment of methylation (Stam et al. 2002). The $b^{\prime}$ locus encodes a transcription factor that regulates genes involved in pigmentation (Chandler and Stam 2004). Two alleles at the $b^{\prime}$ locus, $B-I$ and $B^{\prime}$, result in a dark or light pigmentation phenotype, and this difference corresponds to a higher or lower expression level of each allele, respectively. 
Furthermore, the expression is inversely correlated to different amounts of methylation at seven direct repeats upstream of the gene. When a cross is made between plants containing the $B-I$ and $B^{\prime}$, the methylation at the direct repeat of $B-I$ allele increases and expression decreases, which is subsequently referred to as the paramutated $B^{\prime *}$ allele. $B^{\prime *}$ acts like any $B^{\prime}$ allele because it is able to transmit this methylated state to other $B-I$ alleles. Phenotypically, the consequence of paramutation is that Mendelian segregation is violated because only $B$ (lightly pigmented) progeny are produced from a parent with $B$ $I$ (dark) and $B$ (light) alleles.

Like PAI, the $B^{\prime *}$ is a similar case of trans methylation associated with repeat sequences, although it is distinct from $P A I$ in that once the $B^{\prime *}$ methylation is established it no longer requires the $B^{\prime}$ allele that initiated it to maintain it. This is an important distinction because it shows that epiallelic states can be transmitted independently of the feature that triggers them. This could allow for independent selection on the source and target of a trans-RdDM event. As is the case with fwa, functional components of the RNAi pathway have been shown to be required, indicating that paramutation is likely to be another example of trans RdDM (Erhard et al. 2009; Sidorenko et al. 2009).

A recent study further illustrates how the interplay between structural variation and cis and trans RdDM can affect important traits (Fig. 3). Previously, it had been observed that in a segregating population derived from a Col-0/Shandra (Sha) hybrid cross, one of the four possible homozygous combinations of two unlinked loci, K4 and K5, was significantly underrepresented (Durand et al. 2012). This deviation from expected Mendelian segregation would be consistent with selection against plants where Col-0 is homozygous at K4 and Sha is homozygous at K5. Fine mapping of these regions determined that Sha and Col-0 each have only one expressed folate transporter at K4 and K5, respectively (Durand et al. 2012). Thus, the underrepresented combination lacks a functional copy of the folate transporter gene, which results in a stunted, less fertile plant.
In a surprising twist, however, it was discovered that the Sha accession does in fact contain what appears to be a functional ortholog of AtFolta1 at the K4 locus, but that it is silenced by methylation (Durand et al. 2012). The source of the silencing signal was found to be a rearrangement of an AtFolta-like sequence in Sha at K5 proximal to the functional Sha AtFolta2.

Thus, the Sha AtFolta-like rearrangement appears to silence the Sha AtFolta1 in trans, resulting in the establishment of these incompatible loci (Durand et al. 2012). Further confirmation of this model comes from the observation that, at a low frequency, the Sha AtFolta-like rearrangement can silence the Col-0 AtFolta1 (Durand et al. 2012). Thus, a phenotype that may be an important first step on the road toward speciation is directly attributable to a complex interaction involving gene duplication and rearrangement-induced trans RdDM.

Clearly, repeat sequences and transposons proximal to genes will contribute significantly to natural epigenomic variation because they have been implicated in all examples known to date. As such, variation in the epigenome between accessions is likely to be tightly associated with structural variation. However, a recent pair of papers suggests another potential source of epigenetic variation that is not directly the consequence of changes in primary sequence (Becker et al. 2011; Schmitz et al. 2011). In these two studies, the methylomes were sequenced for a set of five Arabidopsis lines derived from a single ancestor propagated by single seed descent for 30 generations (Shaw et al. 2000). Both studies found that several percent of the methylcytosines were distinct among the descendants when compared to the ancestral lines, and even greater variation existed among the descendants themselves (Becker et al. 2011; Schmitz et al. 2011). Because these lines had previously been sequenced and only a small number of SNPs were observed (average of 30 per line), these large changes in methylation appear to be occurring in the absence of changes in primary sequence (Ossowski et al. 2010). Although most of the differential methylcytosines were found to occur in gene
K4 locus

\section{Col-0 \\ No AtFolta-like sequence found}

K5 locus

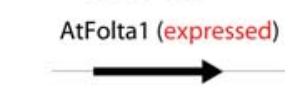

$\mathrm{mC}$
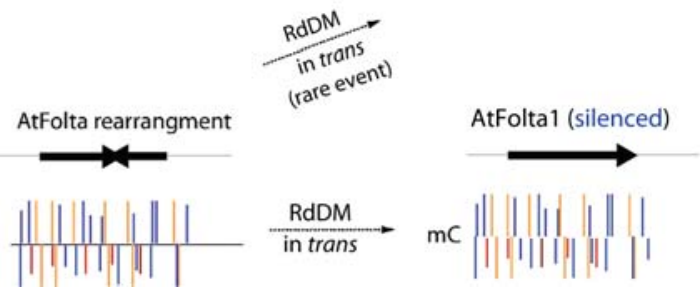

Figure 3. Genetic incompatibility between Col- 0 and Sha caused by genetic and epigenetic variation. An expressed copy of the AtFolta gene is found at the K4 locus in Sha accession (AtFolta2) and at the K5 locus of Col-0 accession (AtFolta1). The Sha accession also contains what appears to be a functional copy of AtFolta1 at K5, but it is silenced by DNA methylation. The source for the silencing signal appears to be a rearranged AtFolta-like sequence at K4 in Sha (solid arrow). In a single $\mathrm{F}_{3}$ of a Col- $0 \times$ Sha cross, the Col- 0 AtFoltal is silenced, likely owing to RdDM in trans originating from the Sha AtFolta rearrangement (doubled arrows). 
bodies, several hundred RdDM loci were found to have diverged in at least one line (Becker et al. 2011; Schmitz et al. 2011). In four cases where the loss of methylation occurred proximal to genes, derepression of silencing was observed, indicating that these stochastic changes do impact transcriptional regulation (Schmitz et al. 2011).

Both studies observed that some of the same variant methylcytosines and methylated loci arose independently in different descendants, suggesting that some of these sites may represent hot spots of epigenetic variation (Becker et al. 2011; Schmitz et al. 2011). Supporting this observation, remethylation was observed at several demethylated loci when an additional generation was tested (Becker et al. 2011). How these rapid changes in the epigenetic landscape in the absence of genetic variation might contribute to longer-term divergence in natural strains is an open question. However, these findings raise the interesting possibility that "epigenetic drift" may be operating as a source of variation on which selection could possibly be acting independent of the genome sequence.

\section{NATURAL EPIGENOMIC VARIATION}

Although sequence variation among divergent Arabidopsis accessions has been evident for many years, it has recently come to light that extensive epigenetic variation also exists in natural plant populations (Vaughn et al. 2007; Groszmann et al. 2011; Greaves et al. 2012b; Shen et al. 2012). Methylome maps generated with nextgeneration bisulfite sequencing for the C24 and Ler by two different groups showed that $\sim 20 \%$ of methylated cytosines are not shared between these two accessions (Greaves et al. 2012b; Shen et al. 2012). Similarly, $\sim 20 \%$ of genomic loci targeted by RdDM were also different between the accessions, indicating substantial variation in the epigenetic landscape that may, in part, be responsible for gene expression variation.

In addition to the Ler and $\mathrm{C} 24$ parents, the methylomes and smRNAomes from reciprocal crosses between the two accessions were also examined by both groups. When crossed, these two accessions show significant heterosis, a phenomenon in which hybrid offspring grow more vigorously than their parents. Both studies explored a potential role of hybrid effects on RdDM on growth. The first study observed a decrease of 24-nucleotide siRNAs in the $F_{1}$ hybrids relative to the parental strains and observed that this corresponded to a decrease in $\mathrm{CHH}$ methylation (Groszmann et al. 2011; Greaves et al. $2012 \mathrm{~b}$ ). These findings suggest that RdDM is compromised in the hybrids relative to their parental strains and the investigators proposed that this could contribute to increased vigor observed in this hybrid because of effects on gene regulation (Groszmann et al. 2011). In the second study, however, no significant change in the 24-nucleotide siRNA levels in the hybrids was observed, and in fact an increase in overall methylation at siRNA loci was reported (Shen et al. 2012). Additionally, they showed that a global decrease in smRNA in the henl background was associated with a loss of heterosis
(Shen et al. 2012). A recent study of siRNA levels in maize hybrids (Barber et al. 2012) observed a decrease in the siRNA levels, similar to Grozzmann et al. (2011). However, in the mopl mutant, which reduces global 24nucleotide siRNA levels, heterosis was unaffected (Barber et al. 2012). This led the authors to conclude that MOP1-specific smRNAs may not play a role in the establishment of hybrid vigor (Barber et al. 2012). It is certainly an interesting hypothesis that epigenomic interactions may have some role in hybrid vigor and, considering the great agronomical value of this poorly understood phenomenon, there will likely be future investigations into this possibility.

\section{CONCLUDING REMARKS}

As we can see from many of the examples of both induced and naturally occurring epialleles, epigenetic variation is tightly linked to sequence and, in particular, transposons and repeats. During the past year, resequenced genomes for 100 Arabidopsis accessions have been published, and although the read size limited the length of de novo assembly possible, there is evidence for substantial structural variation among the accessions (Cao et al. 2011; Gan et al. 2011). For example, Cao et al. (2011) predicted that as many as $80 \%$ of the TAIR 10 annotated transposons are partially or completely deleted within at least one of the 80 sequenced accessions (Cao et al. 2011). Because longer reads at lower cost have been the current trend, it is reasonable to assume that, in the near future, genome assemblies of accessions comparable in quality to the Col-0 reference genome will be available. High-quality assemblies should be extremely informative with regard to how frequently genomic divergence, and in particular structural variation in the form of transposons and repeat features, dictates epigenomic divergence or, alternatively, how frequently epigenomic differences may be attributable to the type of "drift" that is seen in the 30-generations experiments. Finally, complete genome assemblies may allow us to address the frequency, mechanisms, and sequence features underlying RdDM transmission among unlinked homologous sequences, both in individual parents and in hybrid crosses that may potentially be an important source of trait variation displaying non-Mendelian behavior.

\section{REFERENCES}

Ahmed I, Sarazin A, Bowler C, Colot V, Quesneville H. 2011. Genome-wide evidence for local DNA methylation spreading from small RNA-targeted sequences in Arabidopsis. Nucleic Acids Res 39: 6919-6931.

Aufsatz W, Mette MF, van der Winden J, Matzke M, Matzke AJM. 2002. HDA6, a putative histone deacetylase needed to enhance DNA methylation induced by double-stranded RNA. EMBO J 21: 6832-6841.

Aufsatz W, Stoiber T, Rakic B, Naumann K. 2007. Arabidopsis histone deacetylase 6: A green link to RNA silencing. Oncogene 26: 5477-5488.

Barber WT, Zhang W, Win H, Varala KK, Dorweiler JE, Hudson ME, Moose SP. 2012. Repeat associated small RNAs vary 
among parents and following hybridization in maize. Proc Natl Acad Sci 109: 10444-10449.

Bartee L, Malagnac F, Bender J. 2001. Arabidopsis cmt3 chromomethylase mutations block non-CG methylation and silencing of an endogenous gene. Gene Dev 15: 1753-1758.

Becker C, Hagmann J, Müller J, Koenig D, Stegle O, Borgwardt K, Weigel D. 2011. Spontaneous epigenetic variation in the Arabidopsis thaliana methylome. Nature 480: 245-249.

Bender J, Fink GR. 1995. Epigenetic control of an endogenous gene family is revealed by a novel blue fluorescent mutant of Arabidopsis. Cell 83: 725-734.

Bernatavichute YV, Zhang X, Cokus S, Pellegrini M, Jacobsen SE. 2008. Genome-wide association of histone H3 lysine nine methylation with CHG DNA methylation in Arabidopsis thaliana. PLoS One 3: e3156.

Bostick M, Kim JK, Estève P-O, Clark A, Pradhan S, Jacobsen SE. 2007. UHRF1 plays a role in maintaining DNA methylation in mammalian cells. Science 317: 1760-1764.

Brzeski J, Jerzmanowski A. 2002. Deficient in DNA methylation 1 (DDM1) defines a novel family of chromatin-remodeling factors. $J$ Biol Chem 278: 823-828.

Cao X, Jacobsen S. 2002. Role of the Arabidopsis DRM methyltransferases in de novo DNA methylation and gene silencing. Curr Biol 12: 1138-1144.

Cao J, Schneeberger K, Ossowski S, Günther T, Bender S, Fitz J, Koenig D, Lanz C, Stegle O, Lippert C, et al. 2011. Wholegenome sequencing of multiple Arabidopsis thaliana populations. Nat Genet 43: 956-963.

Chan SWL, Zilberman D, Xie Z, Johansen LK, Carrington JC, Jacobsen SE. 2004. RNA silencing genes control de novo DNA methylation. Science 303: 1336.

Chan SWL, Henderson IR, Zhang X, Shah G, Chien JS-C, Jacobsen SE. 2006a. RNAi, DRD1, and histone methylation actively target developmentally important non-CG DNA methylation in Arabidopsis. PLoS Genet 2: e83.

Chan SW-L, Zhang X, Bernatavichute YV, Jacobsen SE. 2006b. Two-step recruitment of RNA-directed DNA methylation to tandem repeats. PLoS Biol 4: e363.

Chandler VL, Stam M. 2004. Chromatin conversations: Mechanisms and implications of paramutation. Nat Rev Genet 5: $532-544$.

Cokus SJ, Feng S, Zhang X, Chen Z, Merriman B, Haudenschild CD, Pradhan S, Nelson SF, Pellegrini M, Jacobsen SE. 2008. Shotgun bisulphite sequencing of the Arabidopsis genome reveals DNA methylation patterning. Nature 452: 215-219.

Cubas P, Vincent C, Coen E. 1999. An epigenetic mutation responsible for natural variation in floral symmetry. Nature 401: $157-161$.

Daxinger L, Kanno T, Bucher E, van der Winden J, Naumann U, Matzke AJM. 2006. A stepwise pathway for biogenesis of 24nt secondary siRNAs and spreading of DNA methylation. EMBO J 28: $48-57$.

Durand S, Bouche N, Strand EP, Loudet O, Camilleri C. 2012. Rapid establishment of genetic incompatibility through natural epigenetic variation. Curr Biol 22: 326-331.

Erhard KF, Stonaker JL, Parkinson SE, Lim JP, Hale CJ, Hollick JB. 2009. RNA polymerase IV functions in paramutation in Zea mays. Science 323: 1201-1205.

Finnegan EJ, Peacock WJ, Dennis ES. 1996. Reduced DNA methylation in Arabidopsis thaliana results in abnormal plant development. Proc Natl Acad Sci 93: 8449-8454.

Fournier-Level A, Korte A, Cooper MD, Nordborg M, Schmitt J, Wilczek AM. 2011. A map of local adaptation in Arabidopsis thaliana. Science 334: 86-89.

Gan X, Stegle O, Behr J, Steffen JG, Drewe P, Hildebrand KL, Lyngsoe R, Schultheiss SJ, Osborne EJ, Sreedharan VT, et al. 2011. Multiple reference genomes and transcriptomes for Arabidopsis thaliana. Nature 477: 419-423.

Greaves I, Groszmann M, Dennis ES, Peacock WJ. 2012a. Trans-chromosomal methylation. Epigenetics 7: 3570-3575.

Greaves IK, Groszmann M, Ying H, Taylor JM, Peacock WJ, Dennis ES. 2012b. Trans chromosomal methylation in Arabidopsis hybrids. Proc Natl Acad Sci 109: 3570-3575.
Groszmann M, Greaves IK, Albertyn ZI, Scofield GN, Peacock WJ, Dennis ES. 2011. Changes in 24-nt siRNA levels in Arabidopsis hybrids suggest an epigenetic contribution to hybrid vigor. Proc Natl Acad Sci 108: 2617-2622.

Hancock AM, Brachi B, Faure N, Horton MW, Jarymowycz LB, Sperone FG, Toomajian C, Roux F, Bergelson J. 2011. Adaptation to climate across the Arabidopsis thaliana genome. Science 334: 83-86.

Hashimoto H, Horton JR, Zhang X, Bostick M, Jacobsen SE, Cheng X. 2008. The SRA domain of UHRF1 flips 5-methylcytosine out of the DNA helix. Nature 455: 826-829.

Henderson IR, Jacobsen SE. 2007. Epigenetic inheritance in plants. Nature 447: 418-424.

Henderson IR, Jacobsen SE. 2008. Tandem repeats upstream of the Arabidopsis endogene SDC recruit non-CG DNA methylation and initiate siRNA spreading. Gene Dev 22: 15971606.

Herr AJ, Jensen MB, Dalmay T, Baulcombe DC. 2005. RNA polymerase IV directs silencing of endogenous DNA. Science 308: $118-120$.

Inagaki S, Kobayashi A, Saze H, Kakutani T. 2009. An Arabidopsis $\mathrm{jmjC}$ domain protein protects transcribed genes from DNA methylation at CHG sites. EMBOJ 28: 1078-1086.

Jackson JP, Lindroth AM, Cao X, Jacobsen SE. 2002. Control of CpNpG DNA methylation by the KRYPTONITE histone H3 methyltransferase. Nature 416: 556-560.

Jeddeloh JA, Bender J, Richards EJ. 1998. The DNA methylation locus DDM1 is required for maintenance of gene silencing in Arabidopsis. Gene Dev 12: 1714-1725.

Jeddeloh JA, Stokes TL, Richards EJ. 1999. Maintenance of genomic methylation requires a SWI2/SNF2-like protein. Nat Genet 22: 94-97.

Johannes F, Porcher E, Teixeira FK, Saliba-Colombani V, Simon M, Agier N, Bulski A, Albuisson J, Heredia F, Audigier P, et al. 2009. Assessing the impact of transgenerational epigenetic variation on complex traits. PLoS Genet 5: e1000530.

Johnson LM, Bostick M, Zhang X, Kraft E, Henderson I, Callis J, Jacobsen SE. 2007. The SRA methyl-cytosine-binding domain links DNA and histone methylation. Curr Biol 17: 379-384.

Johnson LM, Law JA, Khattar A, Henderson IR, Jacobsen SE. 2008. SRA-domain proteins required for DRM2-mediated de novo DNA methylation. PLoS Genet 4: e1000280.

Kankel MW, Ramsey DE, Stokes TL, Flowers SK, Haag JR, Jeddeloh JA, Riddle NC, Verbsky ML, Richards EJ. 2003. Arabidopsis MET1 cytosine methyltransferase mutants. Genetics 163: 1109-1122.

Kanno T, Huettel B, Mette MF, Aufsatz W, Jaligot E, Daxinger L, Kreil DP, Matzke M, Matzke AJM. 2005. Atypical RNA polymerase subunits required for RNA-directed DNA methylation. Nat Genet 37: 761-765.

Kato M, Takashima K, Kakutani T. 2004. Epigenetic control of CACTA transposon mobility in Arabidopsis thaliana. Genetics 168: 961-969.

Kim JK, Estève P-O, Jacobsen SE, Pradhan S. 2009. UHRF1 binds G9a and participates in p21 transcriptional regulation in mammalian cells. Nucleic Acids Res 37: 493-505.

Kinoshita T, Miura A, Choi Y, Kinoshita Y, Cao X, Jacobsen SE, Fischer RL, Kakutani T. 2004. One-way control of FWA imprinting in Arabidopsis endosperm by DNA methylation. Science 303: 521-523.

Kinoshita Y, Saze H, Kinoshita T, Miura A, Soppe WJJ, Koornneef M, Kakutani T. 2007. Control of FWA gene silencing in Arabidopsis thaliana by SINE-related direct repeats. Plant $J$ 49: $38-45$.

Koornneef M, Alonso-Blanco C, Vreugdenhil D. 2004. Naturally occurring genetic variation in Arabidopsis thaliana. Annu Rev Plant Biol 55: 141-172.

Kraft E, Bostick M, Jacobsen S. 2008. ORTH/VIM proteins that regulate DNA methylation are functional ubiquitin E3 ligases. Plant J 56: 704-715.

Law JA, Jacobsen SE. 2010. Establishing, maintaining and modifying DNA methylation patterns in plants and animals. Nat Rev Genet 11: 204-220. 
Lindroth AM, Cao X, Jackson JP, Zilberman D, McCallum CM, Henikoff S, Jacobsen SE. 2001. Requirement of CHROMOMETHYLASE3 for maintenance of CpXpG methylation. Science 292: 2077-2080.

Lindroth AM, Shultis D, Jasencakova Z, Fuchs J, Johnson L, Schubert D, Patnaik D, Pradhan S, Goodrich J, Schubert I, et al. 2004. Dual histone H3 methylation marks at lysines 9 and 27 required for interaction with CHROMOMETHYLASE3. EMBO J 23: 4286-4296.

Lippman Z, Gendrel A-V, Black M, Vaughn MW, Dedhia N, McCombie WR, Lavine K, Mittal V, May B, Kasschau KD, et al. 2004. Role of transposable elements in heterochromatin and epigenetic control. Nature 430: 471-476.

Lister R, O’Malley RC, Tonti-Filippini J, Gregory BD, Berry CC, Millar AH, Ecker JR. 2008. Highly integrated single-base resolution maps of the epigenome in Arabidopsis. Cell 133: $523-536$

Liu X, Yu C-W, Duan J, Luo M, Wang K, Tian G, Cui Y, Wu K. 2012. HDA6 directly interacts with DNA methyltransferase MET1 and maintains transposable element silencing in Arabidopsis. Plant Physiol 158: 119-129.

Malagnac F, Bartee L, Bender J. 2002. An Arabidopsis SET domain protein required for maintenance but not establishment of DNA methylation. EMBO J 21: 6842-6852.

Manning K, Tör M, Poole M, Hong Y, Thompson AJ, King GJ, Giovannoni JJ, Seymour GB. 2006. A naturally occurring epigenetic mutation in a gene encoding an SBP-box transcription factor inhibits tomato fruit ripening. Nat Genet 38: 948-952.

Matsunaga W, Kobayashi A, Kato A, Ito H. 2012. The effects of heat induction and the siRNA biogenesis pathway on the transgenerational transposition of ONSEN, a copia-like retrotransposon in Arabidopsis thaliana. Plant Cell Physiol 53: $824-833$.

Matzke M, Kanno T, Huettel B, Daxinger L, Matzke AJM. 2007. Targets of RNA-directed DNA methylation. Curr Opin Plant Biol 10: 512-519.

Matzke M, Kanno T, Daxinger L, Huettel B, Matzke AJM. 2009. RNA-mediated chromatin-based silencing in plants. Curr Opin Cell Biol 21: 367-376.

Mirouze M, Reinders J, Bucher E, Nishimura T, Schneeberger K, Ossowski S, Cao J, Weigel D, Paszkowski J, Mathieu O. 2009. Selective epigenetic control of retrotransposition in Arabidopsis. Nature 461: 427-430.

Onodera Y, Haag JR, Ream T, Costa Nunes P, Pontes O, Pikaard CS. 2005. Plant nuclear RNA polymerase IV mediates siRNA and DNA methylation-dependent heterochromatin formation. Cell 120: 613-622.

Ossowski S, Schneeberger K, Clark RM, Lanz C, Warthmann N, Weigel D. 2008. Sequencing of natural strains of Arabidopsis thaliana with short reads. Genome Res 18: 2024-2033.

Ossowski S, Schneeberger K, Lucas-Lledó JI, Warthmann N, Clark RM, Shaw RG, Weigel D, Lynch M. 2010. The rate and molecular spectrum of spontaneous mutations in Arabidopsis thaliana. Science 327: 92-94.

Paszkowski J, Grossniklaus U. 2011. Selected aspects of transgenerational epigenetic inheritance and resetting in plants. Curr Opin Plant Biol 14: 195-203.

Reinders J, Wulff BBH, Mirouze M, Mari-Ordonez A, Dapp M, Rozhon W, Bucher E, Theiler G, Paszkowski J. 2009. Compromised stability of DNA methylation and transposon immobilization in mosaic Arabidopsis epigenomes. Gene Dev 23: 939-950.

Richards EJ. 2011. Natural epigenetic variation in plant species: A view from the field. Curr Opin Plant Biol 14: 204-209.

Ronemus MJ, Galbiati M, Ticknor C, Chen J, Dellaporta SL. 1996. Demethylation-induced developmental pleiotropy in Arabidopsis. Science 273: 654-657.

Roudier F, Ahmed I, Bérard C, Sarazin A, Mary-Huard T, Cortijo S, Bouyer D, Caillieux E, Duvernois-Berthet E, Al-Shikhley $\mathrm{L}$, et al. 2011. Integrative epigenomic mapping defines four main chromatin states in Arabidopsis. EMBO J 30: $1928-1938$.
Rozhon W, Baubec T, Mayerhofer J. 2008. Rapid quantification of global DNA methylation by isocratic cation exchange highperformance liquid chromatography. Anal Biochem 375: 354360.

Santuari L, Pradervand S, Amiguet-Vercher A-M, Thomas J, Dorcey E, Harshman K, Xenarios I, Juenger TE, Hardtke CS. 2010. Substantial deletion overlap among divergent Arabidopsis genomes revealed by intersection of short reads and tiling arrays. Genome Biol 11: R4.

Saze H, Mittelsten Scheid O, Paszkowski J. 2003. Maintenance of $\mathrm{CpG}$ methylation is essential for epigenetic inheritance during plant gametogenesis. Nat Genet 34: 65-69.

Saze H, Tsugane K, Kanno T, Nishimura T. 2012. DNA methylation in plants: Relationship to small RNAs and histone modifications, and functions in transposon inactivation. Plant Cell Physiol 53: 766-784.

Schmitz RJ, Schultz MD, Lewsey MG, O'Malley RC, Urich MA, Libiger O, Schork NJ, Ecker JR. 2011. Transgenerational epigenetic instability is a source of novel methylation variants. Science 334: 369-373.

Schneeberger K, Ossowski S, Ott F, Klein JD, Wang X, Lanz C, Smith LM, Cao J, Fitz J, Warthmann N, et al. 2011. Reference-guided assembly of four diverse Arabidopsis thaliana genomes. Proc Natl Acad Sci 108: 10249-10254.

Sharif J, Muto M, Takebayashi S-I, Suetake I, Iwamatsu A, Endo TA, Shinga J, Mizutani-Koseki Y, Toyoda T, Okamura K, et al. 2007. The SRA protein Np95 mediates epigenetic inheritance by recruiting Dnmt1 to methylated DNA. Nature 450: $908-912$.

Shaw RG, Byers DL, Darmo E. 2000. Spontaneous mutational effects on reproductive traits of Arabidopsis thaliana. Genetics 155: 369-378.

Shen H, He H, Li J, Chen W, Wang X, Guo L, Peng Z, He G, Zhong S, Qi Y, et al. 2012. Genome-wide analysis of DNA methylation and gene expression changes in two Arabidopsis ecotypes and their reciprocal hybrids. Plant Cell 24: 875892.

Sidorenko L, Dorweiler JE, Cigan AM, Arteaga-Vázquez M, Vyas M, Kermicle J, Jurcin D, Brzeski J, Cai Y, Chandler VL. 2009. A dominant mutation in mediator of paramutation2, one of three second-largest subunits of a plant-specific RNA polymerase, disrupts multiple siRNA silencing processes. PLoS Genet 5: e1000725.

Slotkin RK, Martienssen R. 2007. Transposable elements and the epigenetic regulation of the genome. Nat Rev Genet 8: $272-285$.

Soppe W, Jacobsen S, Alonso-Blanco C, Jackson J, Kakutani T, Koornneef M, Peeters A. 2000. The late flowering phenotype of fwa mutants is caused by gain-of-function epigenetic alleles of a homeodomain gene. Mol Cell 6: 791-802.

Stam M, Belele C, Dorweiler JE, Chandler VL. 2002. Differential chromatin structure within a tandem array $100 \mathrm{~kb}$ upstream of the maize $b 1$ locus is associated with paramutation. Gene Dev 16: 1906-1918.

Teixeira FK, Heredia F, Sarazin A, Roudier F, Boccara M, Ciaudo C, Cruaud C, Poulain J, Berdasco M, Fraga MF, et al. 2009. A role for RNAi in the selective correction of DNA methylation defects. Science 323: 1600-1604.

To TK, Kim J-M, Matsui A, Kurihara Y, Morosawa T, Ishida J, Tanaka M, Endo T, Kakutani T, Toyoda T, et al. 2011. Arabidopsis HDA6 regulates locus-directed heterochromatin silencing in cooperation with MET1. PLoS Genet 7: e1002055.

Tsukahara S, Kobayashi A, Kawabe A, Mathieu O, Miura A, Kakutani T. 2009. Bursts of retrotransposition reproduced in Arabidopsis. Nature 461: 423-426.

Vaughn MW, Tanurdzić M, Lippman Z, Jiang H, Carrasquillo R, Rabinowicz PD, Dedhia N, McCombie WR, Agier N, Bulski A, et al. 2007. Epigenetic natural variation in Arabidopsis thaliana. PLoS Biol 5: e174.

Vongs A, Kakutani T, Martienssen R, Richards E. 1993. Arabidopsis thaliana DNA methylation mutants. Science 260: 1926-1928.

Weigel D. 2012. Natural variation in Arabidopsis: From molecular genetics to ecological genomics. Plant Physiol 158: 2-22. 
Wierzbicki AT, Ream TS, Haag JR, Pikaard CS. 2009. RNA polymerase $\mathrm{V}$ transcription guides ARGONAUTE4 to chromatin. Nat Genet 41: 630-634.

Woo HR, Pontes O, Pikaard CS, Richards EJ. 2007. VIM1, a methylcytosine-binding protein required for centromeric heterochromatinization. Gene Dev 21: 267-277.

Woo HR, Dittmer TA, Richards EJ. 2008. Three SRA-domain methylcytosine-binding proteins cooperate to maintain global $\mathrm{CpG}$ methylation and epigenetic silencing in Arabidopsis. PLoS Genet 4: e1000156.

Xie Z, Johansen LK, Gustafson AM, Kasschau KD, Lellis AD, Zilberman D, Jacobsen SE, Carrington JC. 2004. Genetic and functional diversification of small RNA pathways in plants. PLoS Biol 2: e104.
Zheng X, Zhu J, Kapoor A, Zhu J-K. 2007. Role of Arabidopsis AGO6 in siRNA accumulation, DNA methylation and transcriptional gene silencing. EMBO J 26: 1691-1701.

Zheng B, Wang Z, Li S, Yu B, Liu JY, Chen X. 2009. Intergenic transcription by RNA polymerase II coordinates Pol IV and Pol V in siRNA-directed transcriptional gene silencing in Arabidopsis. Gene Dev 23: 2850-2860.

Zilberman D, Cao X, Jacobsen SE. 2003. ARGONAUTE4 control of locus-specific siRNA accumulation and DNA and histone methylation. Science 299: 716-719.

Zilberman D, Cao X, Johansen LK, Xie Z, Carrington JC, Jacobsen SE. 2004. Role of Arabidopsis ARGONAUTE4 in RNAdirected DNA methylation triggered by inverted repeats. Curr Biol 14: 1214-1220. 


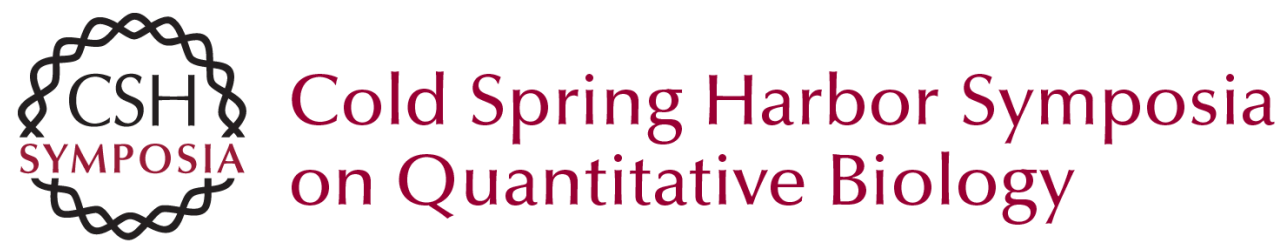

\section{Epiallelic Variation in Arabidopsis thaliana}

R.C. O'Malley and J.R. Ecker

Cold Spring Harb Symp Quant Biol 2012 77: 135-145 originally published online December 5, 2012

Access the most recent version at doi:10.1101/sqb.2012.77.014571

References This article cites 94 articles, 36 of which can be accessed free at: http://symposium.cshlp.org/content/77/135.full.html\#ref-list-1

\section{License}

Email Alerting

Receive free email alerts when new articles cite this article - sign up in

Service the box at the top right corner of the article or click here. 